\section{ARRHYTHMIAS}

\section{Early mortality after AF ablation}

Early procedure-related mortality affects almost 1 in 200 patients undergoing catheter ablation for atrial fibrillation (AF), according to a new study published in the Journal of the American College of Cardiology. Notably, this rate of postprocedural death exceeds the mortality reported in major randomized, controlled clinical trials.

Catheter ablation is an increasingly used therapeutic strategy for the management of $\mathrm{AF}$ and has been shown in numerous major clinical trials such as CASTLE-AF and CABANA to be both effective and safe. No procedure-related deaths were reported in these trials; however, real-world data on early mortality after catheter ablation for $\mathrm{AF}$ are scarce. To address this knowledge gap, Cheng and colleagues used data from an all-payer, nationally representative US-based database to assess 30-day mortality and complications linked with catheter ablation for the treatment of AF. Among 60,203 patients who underwent the procedure, $276(0.46 \%)$ patients died early after $\mathrm{AF}$ ablation, with $54.3 \%$ of these deaths occurring during hospital readmission within 30 days of the procedure. Quarterly postprocedural mortality increased from $0.25 \%$ to $1.35 \%$ between 2010 and 2015 . Advanced age and a higher burden of comorbidities including coronary artery disease, congestive heart failure (CHF) and chronic lung or kidney disease were associated with higher risk of postprocedural death. After adjustment for age and comorbidities, patients who had procedural complications or underwent $\mathrm{AF}$ ablation at low-volume centres (defined as hospitals with a case volume of $<21$ ablations annually) were more likely to die early after AF ablation.

"It should be emphasized that the benefits of [AF] ablation for patients with CHF shown in recent clinical trials have largely involved high-volume academic centres, whose outcomes may not be replicated by lower-volume operators and centres in the real world," explain the investigators. "Therefore, as more patients with $\mathrm{CHF}$ undergo $[\mathrm{AF}]$ ablation, the rates of early mortality after the procedure may continue to rise on a national level."

Karina Huynh

ORIGINAL ARTICLE Cheng, E. P. et al. Risk of mortality following catheter ablation of atrial fibrillation. J. Am. Coll.

Cardiol. 74, 2254-2264 (2019)

$\Rightarrow$ HYPERTENSION

\title{
Blood-pressure medication timing matters
}

Current hypertension management guidelines do not specify a preferred time for treatment with blood pressure (BP)-lowering medications, but the most common recommendation by clinicians is morning treatment. However, new findings from the Hygia Chronotherapy trial suggest that taking antihypertensive medications at bedtime is associated with better BP control and a 45\% reduction in the risk of cardiovascular disease (CVD) compared with taking the medication in the morning upon waking up.

This multicentre, controlled, prospective trial was conducted in the primary care setting and included 19,084 patients with hypertension $(10,614$ men and 8,470 women) who had a prescription of more than one BP-lowering drug. Patients were randomly assigned to take their entire daily dose of antihypertensive medications either at bedtime or upon waking up. Ambulatory BP (ABP) monitoring for $48 \mathrm{~h}$ was performed at baseline and at every scheduled clinic visit. After a median of 6.3 years, the bedtime-treatment group had improved ABP control, with lower asleep systolic BP (SBP) and greater sleep-time relative SBP decline (that is, BP dipping), compared with the morning-treatment group. Furthermore, the primary CVD outcome (a composite of CVD death, myocardial infarction, coronary revascularization, heart failure or stroke) occurred in fewer patients in the bedtime-treatment group than in the morning-treatment group (HR 0.55, 95\% CI $0.50-0.61, P<0.001)$ after adjustment for factors including age, sex, asleep SBP mean, sleep-time relative SBP decline and previous CVD. Analysis of the individual primary outcome components showed similar results.

In summary, the time of treatment seems to be important for BP control and protection against CVD. However, the mechanism for the benefit of bedtime dosing is unclear. Ongoing, large-scale trials assessing antihypertensive treatment timing might provide further insights into the role of circadian treatment patterns.

Irene Fernández-Ruiz

ORIGINAL ARTICLE Hermida, R. C. et al. Bedtime hypertension treatment improves cardiovascular risk reduction: the Hygia Chronotherapy Trial. Eur. Heart J. https://doi.org/10.1093/ eurheartj/ehz754 (2019)

\section{DYSLIPIDAEMIA}

\section{Benefit of early initiation of statins for $\mathrm{FH}$}

Statin therapy initiated during childhood in patients with familial hypercholesterolaemia $(\mathrm{FH})$ slows the rate of carotid intimamedia thickening and reduces the risk of cardiovascular events and death. This finding comes from a 20-year follow-up of patients who had previously participated in a 2-year randomized trial of pravastatin therapy.

A total of 214 patients with FH (genetically confirmed in $98 \%$ ) who had previously participated in the trial and 95 of their unaffected siblings were invited for follow-up. Overall, $79 \%$ of the patients with $\mathrm{FH}$ reported continued use of lipid-lowering medication.

Among the patients with $\mathrm{FH}$, the mean LDL-cholesterol level had decreased by $32 \%$ from $237.3 \mathrm{mg} / \mathrm{dl}$ at baseline to $160.7 \mathrm{mg} / \mathrm{dl}$ at follow-up. By contrast, in the unaffected siblings, the mean LDL-cholesterol level had increased by $24 \%$ from $98.5 \mathrm{mg} / \mathrm{dl}$ at baseline to $121.9 \mathrm{mg} / \mathrm{dl}$ at follow-up. Mean carotid intimamedia thickness was greater in patients with $\mathrm{FH}$ than in their unaffected siblings at baseline $(0.446 \mathrm{~mm}$ versus $0.439 \mathrm{~mm})$. At the 20 -year follow-up, the mean carotid intima-media thickness was $0.555 \mathrm{~mm}$ in patients with $\mathrm{FH}$ and $0.551 \mathrm{~mm}$ in their unaffected siblings, indicating a mean rate of thickening of $0.0056 \mathrm{~mm}$ per year and $0.0057 \mathrm{~mm}$ per year in each group, respectively.

The cumulative incidence of cardiovascular events and death from cardiovascular causes by the age of 39 years was $1 \%$ and $0 \%$, respectively, in patients with $\mathrm{FH}$. By contrast, among 156 of their parents with $\mathrm{FH}$ (who had not received statin therapy from an early age), the equivalent rates were $26 \%$ and $7 \%$, respectively.

These findings support the notion that atherogenesis is the product of both the magnitude and the duration of exposure of the arterial wall to LDL cholesterol and reinforce clinical guideline recommendations to initiate statin therapy by the age of $8-10$ years in patients with $\mathrm{FH}$

Gregory B. Lim

ORIGINAL ARTICLE Luirink, I. K. et al. 20-year follow-up of statins in children with familial hypercholesterolemia. N. Engl. J. Med. 381, 1547-1556 (2019)

RELATED ARTICLE Berberich, A. J. \& Hegele, R. A. The complex molecular genetics of familial hypercholesterolaemia. Nat. Rev. Cardiol. 16, 9-20 (2019) 